\title{
Matroid Online Bipartite Matching and Vertex Cover
}

YAJUN WANG, Microsoft Corporation

SAM CHIU-WAI WONG, UC Berkeley

The Adwords and Online Bipartite Matching problems have enjoyed a renewed attention over the past decade due to their connection to Internet advertising. Our community has contributed, among other things, new models (notably stochastic) and extensions to the classical formulations to address the issues that arise from practical needs. In this paper, we propose a new generalization based on matroids and show that many of the previous results extend to this more general setting. Because of the rich structures and expressive power of matroids, our new setting is potentially of interest both in theory and in practice.

In the classical version of the problem, the offline side of a bipartite graph is known initially while vertices from the online side arrive one at a time along with their incident edges. The objective is to maintain a decent approximate matching from which no edge can be removed. Our generalization, called Matroid Online Bipartite Matching, additionally requires that the set of matched offline vertices be independent in a given matroid. In particular, the case of partition matroids corresponds to the natural scenario where each advertiser manages multiple ads with a fixed total budget.

Our algorithms attain the same performance as the classical version of the problems considered, which are often provably the best possible. We present $1-1 / e$-competitive algorithms for Matroid Online Bipartite Matching under the small bid assumption, as well as a $1-1 / e$-competitive algorithm for Matroid Online Bipartite Matching in the random arrival model. A key technical ingredient of our results is a carefully designed primal-dual waterfilling procedure that accommodates for matroid constraints. This is inspired by the extension of our recent charging scheme for Online Bipartite Vertex Cover.

Finally, given that only few online problems were studied in the submodular fashion, the techniques introduced in this paper for tackling submodularity in the online setting may be of independent interest.

Additional Key Words and Phrases: Primal-dual; matching; vertex cover

\section{INTRODUCTION}

Recent years have seen an explosion of research on various online matching problems thanks to Internet advertising. One prominent example is Online Bipartite $b$-matching where advertisers wish to advertise on a search engine platform such as Google or Bing. Each ad has a budget which specifies the maximum number of times it should be displayed to impressions arriving in an online fashion. Upon the arrival of an impression, the search engine must decide on which ad to display, if any, while respecting the budget constraint. The goal is to display as many ads as possible to maximize revenue.

This simple setting encapsulates the essence of many online allocation problems. Nevertheless, its lack of sophistication means that if used directly, it could be a poor model for specific applications. Recent research has responded by proposing variants of the problem driven by practical needs. Examples include different stochastic models (e.g. [Feldman et al. 2009b; Goel and Mehta 2008]) and the free disposal model [Feld-

This research was supported by NSF grants CCF0964033 and CCF1408635, and by Templeton Foundation grant 3966.

Permission to make digital or hard copies of all or part of this work for personal or classroom use is granted without fee provided that copies are not made or distributed for profit or commercial advantage and that copies bear this notice and the full citation on the first page. Copyrights for components of this work owned by others than ACM must be honored. Abstracting with credit is permitted. To copy otherwise, or republish, to post on servers or to redistribute to lists, requires prior specific permission and/or a fee. Request permissions from permissions@acm.org.

EC'16, July 24-28, 2016, Maastricht, The Netherlands.

ACM 978-1-4503-3936-0/16/07 ...\$15.00.

Copyright is held by the owner/author(s). Publication rights licensed to ACM.

http://dx.doi.org/10.1145//2940716.2940793 
man et al. 2009a]. In this paper, we observe and address a new limitation which may be of real world interest especially for Internet advertising.

Most of these models have the shortcoming that the budget for each ad is specified independently. Many advertisers, especially those selling real products, are typically hosting multiple ads. Under the simplistic setting above, he would be able to only budget for each ad independently. This would not be ideal as his ads may be correlated, meaning that his budget for one ad may depend on how much he spends on another ad. For instance, suppose that a soft drink distributor is advertising for both coke and sprite. He is willing to spend $\$ 5$ on coke, $\$ 4$ on sprite but only $\$ 8$ on both. Under the current framework, this kind of preference is impossible to represent by two separate budgets for coke and sprite.

We address this limitation by allowing an advertiser to specify the budget $f(S)$ for each subset $S$ of his ads, with one mild restriction. We require that $f$ be monotone submodular. The search engine would then display ads from $S$ at most a total of $f(S)$ times. We argue that the assumption on $f$ is only mild. Monotonicity is clearly reasonable. Submodularity also makes sense as one should expect to observe diminishing marginal returns for the similar ads hosted by the advertiser. Returning to our example on coke and sprite, if $\$ 5$ is already spent on coke, our advertiser may think that the soft drink market is more saturated than before and hence spend less on sprite $(\$ 3)$ than he otherwise would $(\$ 4)$.

Readers well-versed in matroid theory would recognize that this is equivalent to imposing a (poly)matroid constraint on the set of ads. Somewhat surprisingly, many of the existing results in the literature conform very well to this matroid generalization and we are able to obtain algorithms with the same performance in this considerably more general setting.

From a practical standpoint, we believe that this is a desirable feature to be introduced to Internet advertising. Because of the need to budget individually, advertisers of multiple ads may currently be under-budgeting to avoid overspending. In the soft drink example, the distributor may submit a budget of $f($ coke $)=\$ 4$ and $f($ sprite $)=\$ 4$. This would be suboptimal if he ends up exhausting his $\$ 4$ budget on coke but spending only $\$ 1$ on coke. Needless to say, this would not be efficient for the search engine either as additional revenue could be gained from these potential ads display had the advertisers been given greater flexibility. We believe that our matroid generalization may open up window of opportunities to create values for both ad platforms and advertisers.

Having motivated for the new matroid constraint, we formally define the problems studied in this paper and our results. We note that many of the other works in the literature also generalize readily to the matroid setting but to avoid being repetitious, we have chosen only a few representative problems to illustrate how we cope with the additional matroid constraint without sacrificing performances.

\subsection{Our results and techniques}

We present $1-1 / e$-competitive algorithms for all of the problems considered in this paper. As we have alluded, our purpose is to illustrate how the seemingly much more general matroid constraint can be incorporated into existing works without any loss in competitive ratio. To this end, we have selected two representative problems and show rigorously that their matroid generalizations still admit $1-1 / e$-competitive algorithms. Specifically, the design and analysis of our new algorithms make use of the charging scheme of [Wang and Wong 2015] and the primal-dual frameworks of [Buchbinder et al. 2007; Devanur et al. 2013b].

To the best of our knowledge, this is the first time that the primal-dual analysis is applied to an online problem which involves submodularity. The closest example in 
online matching that we are aware of is [Devanur and Jain 2012], which involves continuous concave functions rather than discrete submodular functions. We hope that the primal-dual analysis method will emerge as a powerful tool for tackling submodularflavored online problems.

The new results in this paper are summarized below.

- Optimal waterfilling $1-1$ /e-competitive algorithm for Matroid Online Bipartite Vertex Cover and Matching (in the adversarial model) under the small bid assumption.

- Greedy is 1-1/e-competitive algorithm for Matroid Online Bipartite Matching in the random arrival Model (without the small bid assumption).

All of the above algorithms are greedy in nature and hence simple to implement. In addition to existing ideas in the literature, the design and analysis of our algorithms introduce the following new techniques and machineries.

Two dimensional charging scheme. The recent work of [Wang and Wong 2015] introduces a (single-dimensional) charging scheme-based analysis for online bipartite vertex cover. To tackle submodularity, we propose a new charging scheme that operates on the so-called "bar chart diagrams" for the Lovasz extension. The new scheme is based on a two-dimensional charging function of the bar chart diagram. Our analysis is therefore much more involved than the original scheme.

Convex programming duality. The primal-dual framework [Buchbinder et al. 2007; Devanur et al. 2013b] is one of the most powerful techniques for attacking various online matching problems, with almost all of the existing works based on LP duality. To address matroid constraint one must however resort to convex programming (or equivalently, LPs with exponentially many variables or constraints). Our contribution is demonstrating that the existing machineries for handling matroids and submodularity in the offline optimization setting (e.g. Lovasz extension and base polyhedrons) conform seamlessly to the online primal-dual framework. The main issue here is understanding why the diminishing return property of submodular functions is compatible with the algorithms for online bipartite matching. Although it is hard to explain without referring to the specific details of the algorithms, fundamentally everything works out nicely in this paper because each "linear component" of the Lovasz extension is compatible with the online primal-dual analysis. This point will become clear when we analyze our algorithms.

\subsection{Previous works}

Karp et al. [Karp et al. 1990] gave the optimal $1-1 / e$-competitive algorithm for the online bipartite matching problem. Different variants of it have been extensively studied. These include $b$-matching [Kalyanasundaram and Pruhs 2000], vertex-weighted version [Aggarwal et al. 2011; Devanur et al. 2013b], adwords [Buchbinder et al. 2007; Devenur and Hayes 2009a; Mehta et al. 2007; Devanur et al. 2013b; Devanur and Jain 2012; Goel and Mehta 2008; Aggarwal et al. 2011; Devanur et al. 2012; Devenur and Hayes 2009b] and online market clearing [Blum et al. 2006].

To get around the worst-case analysis, other research directions study the problem with weaker adversarial models by assuming stochastic inputs [Feldman et al. 2009b; Manshadi et al. 2011; Mahdian and Yan 2011; Karande et al. 2011] as well as general graphs [Bansal et al. 2010].

Organization. The rest of this paper is structured as follows. In Section 2, we introduce various notions and tools from combinatorial optimization needed for our results. Section 3 is on the basic online bipartite vertex cover problem and introduces the charging scheme from [Wang and Wong 2015]. Section 4 studies matroid online 
bipartite vertex cover and builds on the previous charging scheme. It also includes an alternate primal-dual analysis of the algorithm which proves our result on matroid online bipartite matching. Finally, Section 5 is on matroid online bipartite matching in the random arrival model. We conclude in Section 6 with some open problems.

\section{PRELIMINARIES}

We first introduce some standard notions in combinatorial optimization before stating our problems. In this paper we consider only undirected bipartite graphs $G=(L, R, E)$, where $L$ and $R$ are the sets of left and right vertices respectively.

Given $G=(L, R, E)$, a vertex cover (VC) of $G$ is a subset of vertices $C \subseteq L \cup R$ such that for each edge $(u, v) \in E, C \cap\{u, v\} \neq \emptyset$. A matching of $G$ is a subset of edges $M \subseteq E$ such that each vertex $u \in L, v \in R$ is incident to at most one edge in $M$. The size of a vertex cover $C$ and a matching $M$ is just $|C|$ and $|M|$.

$(\mathbf{y}, \mathbf{z}) \in\left([0,1]^{L},[0,1]^{R}\right)$ is a fractional vertex cover if for any edge $(u, v) \in E, y_{u}+z_{v} \geq 1$. $\mathbf{x} \in[0,1]^{E}$ is a fractional matching if for each $u \in L, x_{u}:=\sum_{v \in N(u)} x_{u v} \leq 1$ and for each $v \in R, x_{v}:=\sum_{u \in N(v)} x_{u v} \leq 1$. The size of a fractional vertex cover $(\mathbf{y}, \mathbf{z})$ and a fractional matching $M$ is just $\sum_{u \in L} y_{u}+\sum_{v \in R} z_{v}$ and $\sum_{e \in E} x_{e}=\sum_{u \in L} x_{u}=\sum_{v \in R} x_{v}$. We call $y_{u}$ the potential of $u$. It is well-known that VC and matching are dual of each other in bipartite graphs.

A set function $f: 2^{L} \longrightarrow \mathbb{R}$ is said to be submodular if for all $A, B \subseteq L$,

$$
f(A)+f(B) \geq f(A \cup B)+f(A \cap B) .
$$

One often finds the following equivalent definition useful: for every $A, B \subseteq L$ with $A \subseteq B$ and every $e \in L$ (using the shorthand $A+e=A \cup\{e\}$ ),

$$
f(A+e)-f(A) \geq f(B+e)-f(B) .
$$

Loosely speaking, this says that the marginal return of adding an element $e$ to a larger set is smaller. This property makes submodular functions appealing beyond its mathematical beauty as this phenomenon is observed in many real-life scenarios, especially those which arise from economic settings.

A submodular function $f$ is monotone if $f(B) \geq f(A)$ for every $A, B \subseteq L$ with $A \subseteq B$.

Given a nonnegative ${ }^{1}$ monotone submodular function $f(\cdot), \mathrm{x} \in[0,1]^{E}$ is a matroid matching defined by $f$ if for all $v \in R$,

$$
x_{v}:=\sum_{u \in N(v)} x_{u v} \leq 1
$$

and for all $S \subseteq L$,

$$
x_{S}:=\sum_{u \in S} x_{u}=\sum_{u \in S} \sum_{v \in N(u)} x_{u v} \leq f(S) .
$$

It is easy to see that this is indeed a generalization of the usual matching which corresponds to $f(S)=|S|$.

The rest of this section formally defines our problems and introduces the machineries needed to tackle our problems.

\subsection{Problem statements}

In all of these problems, the underlying graph is bipartite $G=(L, R, E)$ with offline vertices $L$. The right vertices $R$ arrive online one by one. When a vertex $v \in R$ arrives, all of its incident edges are revealed.

\footnotetext{
${ }^{1}$ Our results actually still hold even if $f(S)<0$ for some $S \subseteq L$, in which case we can just remove $S$ as its vertices can never be matched.
} 
- Online bipartite vertex cover (OBVC) The algorithm must maintain at all time a valid monotone vertex cover $C$, i.e. no vertex can ever be removed from $C$ once it is put into $C$. Thus the algorithm essentially decides whether to assign $v$ or $N(v)$ to $C$ upon the arrival of an online vertex $v$. The objective is to minimize the size of $C$ in the end.

- Matroid online bipartite vertex cover (MOBVC) The setting is exactly identical to OBVC except that the objective function is $f(C \cap L)+|C \cap R|$ instead of $|C|$. Here $f(\cdot)$ is a nonnegative monotone submodular function. OBVC is a special case of MOBVC in which $f$ is simply the cardinality function $f(S)=|S|$.

- Matroid online bipartite (fractional) matching (MOBM) The setting of MOBM is similar to OBVC. Here we have a nonnegative monotone submodular function $f(\cdot)$ on the left vertices and the algorithm maintains a matroid matching $\mathrm{x}$ instead of a vertex cover. When an online vertex $v$ arrives, the algorithm must initialize all $x_{u v}$ for $u \in N(v)$ so that $\mathbf{x}$ is still a valid matroid matching. The objective is to maximize the size of $\mathbf{x}$, i.e. $\sum_{e \in E} x_{e}=\sum_{u \in L} x_{u}=\sum_{v \in R} x_{v}$. The integral version of MOBM has all $x_{e} \in\{0,1\}$ and $f$ being a matroid rank function, which is integer-valued and nonnegative monotone submodular.

Finally, although not needed for this paper, we remark that the offline versions of these problems can be solved in polynomial time by matroid intersection.

Fractional vs. Integral. As with the previous waterfilling algorithms for online bipartite matching, the integral version with small bid assumption is equivalent to the fractional version [Buchbinder et al. 2007; Devanur et al. 2013a]. For the sake of convenience we present our waterfilling algorithm for MOBM in the fractional setting.

\subsection{Lovasz extension of submodular functions}

Given a submodular function $f: 2^{L} \longrightarrow \mathbb{R}$, the Lovasz extension $\hat{f}:[0,1]^{L} \longrightarrow \mathbb{R}$ is a continuous convex relaxation of $f$ and is defined by

$$
\hat{f}(\mathbf{y})=\mathbb{E}_{t}[f(L(t))]
$$

where $L(t)=\left\{u \in L: y_{u} \geq t\right\}$ and the expectation is taken over $t$ chosen uniformly at random from $[0,1]$. It is easy to check one does have $f(S)=\hat{f}\left(I_{S}\right)$. Here $I_{S}$ is the indicator variable for $S \subseteq L$.

We will also make heavy use of an equivalent definition in this paper. Given $\mathbf{y} \in$ $[0,1]^{L}$, order the vertices of $L=\{1,2, \ldots, n\}$ in such a way that $0=y_{0} \leq y_{1} \leq y_{2} \leq \ldots \leq$ $y_{n} \leq y_{n+1}=1$. Let $Y_{i}=\{i, i+1, \ldots, n\}$ and $Y_{n+1}=\emptyset$. Then

$$
\hat{f}(\mathbf{y})=\sum_{i=1}^{n+1}\left(y_{i}-y_{i-1}\right) f\left(Y_{i}\right) .
$$

An immediate implication of this formulation is that by restricting $\mathbf{y} \in[0,1]^{L}$ to some fixed ordering $\sigma:\{1,2, \ldots,|L|\} \longrightarrow L, \hat{f}(\mathbf{y})$ is a linear function. This property will be used in various places of this paper.

Finally, note that for monotone submodular function $f$, its Lovasz extension $\hat{f}(\mathbf{y})$ is monotonically increasing (in each coordinate).

In the next section, we introduce a bar chat interpretation of the Lovasz function. This representation plays an important role in analyzing MOBVC and MOBM. 
Table I.

\begin{tabular}{|c|c|c|c|}
\hline Primal: & & Dual: & \\
\hline s.t. & $\begin{array}{l}\max \sum_{e \in E} x_{e} \\
x_{v} \leq 1, \forall v \in R \\
x_{S} \leq f(S), \forall S \subseteq L \\
\mathbf{x}>0\end{array}$ & s.t. & $\begin{array}{l}\min \hat{f}(\mathbf{y})+\sum_{v \in R} z_{v} \\
y_{u}+z_{v} \geq 1, \forall(u, v) \in E \\
\mathbf{y}, \mathbf{z} \geq 0\end{array}$ \\
\hline
\end{tabular}

2.2.1. Bar-chart representation. Given $\mathbf{y} \in[0,1]^{L}$, the bar chart representation of $\hat{f}(\mathbf{y})$ is the set

$$
\bigcup_{t \in[0,1]}\{t\} \times[0, f(L(t))] .
$$

Notice that the bars are decreasing in height as $t$ increases because $f$ is monotone. If we order $L=\{1,2, \ldots, n\}$ in such a way that $y_{1} \leq y_{2} \leq \ldots \leq y_{n}$. Using the notation in the last section, the bar chart representation consists of the bars

$$
\left[0, y_{1}\right] \times\left[0, f\left(Y_{1}\right)\right],\left[y_{1}, y_{2}\right] \times\left[0, f\left(Y_{2}\right)\right], \ldots,\left[y_{n-1}, y_{n}\right] \times\left[0, f\left(Y_{n}\right)\right],\left[y_{n}, 1\right] \times\left[0, f\left(Y_{n+1}\right)\right] .
$$

This is often a useful way to visualize the Lovasz extension $\hat{f}(\mathbf{y})=\sum_{i=1}^{n+1}\left(y_{i}-y_{i-1}\right) f\left(Y_{i}\right)$ as each term in the summand corresponds to precisely a bar in the bar-chart representation. In particular, $\hat{f}(\mathbf{y})$ is the area of the bar chart.

Strictly speaking, a bar can be empty (e.g. when $y_{i}=y_{i+1}$ ) but we shall implicitly disregard them hereafter as it does not affect our proofs in any way and would only make the notations more cumbersome.

Readers may find that it is sometimes more intuitive to view the bar chart as the function $t \mapsto f(L(t))$ for $t \in[0,1]$. Figure 1 shows a bar chart representation with 5 bars, of which the last one corresponds to the empty set and has height $f(\emptyset)=0$.

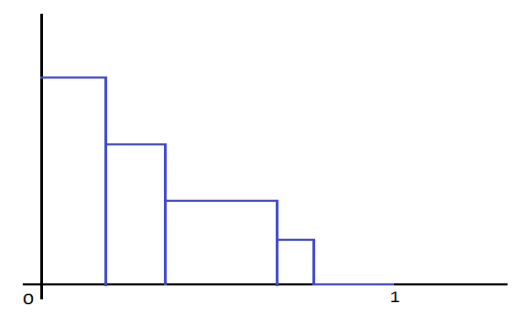

Fig. 1. Bar chart representation of Lovasz extension

\subsection{Convex program for bipartite matroid matching and vertex cover}

Recall the notations $x_{v}:=\sum_{u \in N(v)} x_{u v}$ and $x_{S}:=\sum_{u \in S} x_{u}:=\sum_{u \in S} \sum_{v \in N(u)} x_{u v}$. The primal and dual convex programs below are used in the primal-dual analysis of our algorithms for MOBM and MOBVC.

Readers who familiar with polymatroid intersection should recognize that the primal is actually the polytope associated with the intersection of a partition matroid on $R$ and a polymatroid on $L$ defined by the submodular function $f$.

As in the usual primal-dual method, weak duality ${ }^{2}$ is required in order to bound the size of the primal and dual solutions. A proof is in the full version.

\footnotetext{
${ }^{2}$ In fact, even strong duality holds but this is not needed for our analysis.
} 
Lemma 2.1. (weak duality) For any feasible solutions $\mathbf{x}$ and $(\mathbf{y}, \mathbf{z})$ to the primal and dual programs above, we have

$$
\sum_{e \in E} x_{e} \leq \hat{f}(\mathbf{y})+\sum_{v \in R} z_{v} .
$$

2.4. Rounding scheme for online bipartite vertex cover

For a fractional vertex cover $(\mathbf{y}, \mathbf{z})$ in an online algorithm for the online bipartite vertex cover problems studied in this paper, we can always round it to an integral solution by following simple scheme. We first sample $\gamma$ uniformly at random from [0,1]. Afterwards, for any vertex $u \in L$, we place $u$ in the cover as long as $y_{u} \geq \gamma$. On the other hand, for any vertex $v \in R$, we place $v$ in the cover when $z_{v} \geq 1-\gamma$. It is not hard to verify that this rounding scheme indeed maintains a monotone vertex cover since $(\mathbf{y}, \mathbf{z})$ is monotone.

Now consider an algorithm for the matroid online bipartite vertex cover problem, with fractional solution $(\mathbf{y}, \mathbf{z})$. Let $C(\gamma)$ be the vertices in the integral cover after the rounding with $\gamma$. The performance of our algorithm with this rounding scheme is

$$
\mathbb{E}_{\gamma}[f(C(\gamma) \cap L)]+\mathbb{E}_{\gamma}[|C(\gamma) \cap R|]=\hat{f}(\mathbf{y})+\sum_{v \in R} z_{v} .
$$

Therefore, this rounding scheme does not incur a loss for MOBVC. Since OBVC is a special case of MOBVC, the rounding scheme also works for OBVC.

\section{5. $\alpha$ and two integrals}

To simplify our notation, we denote the optimal competitive ratio for OBVC as

$$
1+\alpha:=\frac{1}{1-1 / e}
$$

throughout this paper. In our analyses, the following two integrals will often be useful.

$$
\int_{0}^{1} \frac{1-t}{t+\alpha} d t=\alpha, \quad \int_{0}^{1} \frac{1}{t+\alpha} d t=1
$$

\section{ONLINE BIPARTITE VERTEX COVER}

In this section, we present the algorithm GreedyAlloction for OBVC from [Wang and Wong 2015] as well its charging-based analysis. They will be the corner-stone of our new results.

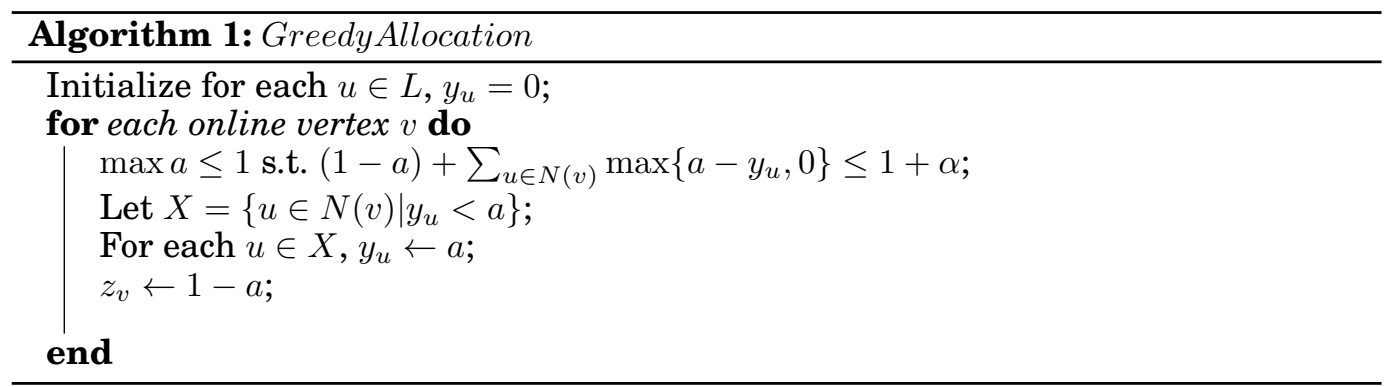

When an online vertex $v$ arrives, we can choose to place $v$ in the cover which has a cost of 1 . Alternately, we can put all the vertices from $N(v)$ into the cover. In 
GreedyAllocation, we attempt to put as much $N(v)$ into the cover with a resource constraint of $1+\alpha$. GreedyAllocation is greedy in the sense that we try to make $a$, i.e. the potential on $N(v)$, as large as possible.

Now we present the charging-based analysis of GreedyAllocation from [Wang and Wong 2015]. Let $C^{*}$ be a minimum vertex cover of $G$. We will charge the potential increment to vertices of $C^{*}$ so that each vertex of $C^{*}$ is charged at most $1+\alpha$.

Given an online vertex $v$, we consider the following two cases.

(1) $v \in C^{*}$. In this case, we charge the potential increment in $N(v)$ and $v$ in the algorithm to $v$. In particular, $v$ will be charged at most $1+\alpha$.

(2) $v \notin C^{*}$ which implies $N(v) \subseteq C^{*}$. In this case, $N(v)$ should take the potential increment on themselves as well as $y_{v}=1-a$ used by $v$. The following charging scheme is critical. Intuitively, if $\sum_{u \in X}\left(a-y_{u}\right)=a+\alpha$, we should charge $\frac{1-a}{a+\alpha}\left(a-y_{u}\right)$ to $u \in X$ since the fair "unit charge" is $\frac{1-a}{a+\alpha}$. Because $\frac{1-a}{a+\alpha}$ is decreasing in $a, \frac{1-a}{a+\alpha}\left(a-y_{u}\right)$ can be upper bounded by

$$
\int_{y_{u}}^{a} \frac{1-t}{t+\alpha} d t
$$

The next lemma indicates that the total charge is sufficient.

LEMMA 3.1. [Wang and Wong 2015] Let $F(x)=\int_{0}^{x} \frac{1-t}{t+\alpha} d t$. If $\sum_{u \in X}\left(a-y_{u}\right)=a+\alpha$ for some set $X$ and $a \geq y_{u}$ for $u \in X$, then

$$
1-a \leq \sum_{u \in X}\left(F(a)-F\left(y_{u}\right)\right) .
$$

The lemma implies that $v \notin C^{*}$ can be charged at most $1+F(1)-F(0)=1+\int_{0}^{1} \frac{1-t}{t+\alpha} d t=$ $1+\alpha$. By the previous discussion, we then have:

THEOREM 3.2. [Wang and Wong 2015] GreedyAllocation is $1+\alpha$-competitive for (fractional) $O B V C$.

As mentioned in Section 2.4, it is possible to convert any online fractional vertex cover algorithms while preserving the competitive ratio in expectation.

\section{MATROID ONLINE BIPARTITE MATCHING AND VERTEX COVER}

As in OBVC, we first consider the fractional version of MOBVC. Our objective is then to minimize $\hat{f}(\mathbf{y})+\sum_{v \in R} z_{v}$, which is a convex relaxation of $f(C \cap L)+|C \cap R|$. At the end of our analysis, we will show that it is possible to round our solution to an integral VC with the same size in expectation. Thus our algorithm for fractional MOBVC also works for integral MOBVC.

Our algorithm for MOBVC is still greedy. The analysis, however, relies on a "twodimensional" charging scheme in which the new additional regions of the bar chart representation (introduced in Section 2.2.1) are charged. We will see that the previous charging scheme for OBVC is a simplistic version of this more sophisticated scheme.

We also give an alternate primal-dual analysis of our algorithm which will imply a corresponding result for MOBM as a by-product. Our method builds on the previous scheme [Buchbinder et al. 2007] for online bipartite matching (and effectively OBVC).

Since the primal-dual analysis implies both the results on MOBM and MOBVC, the charging analysis may seem redundant. We stress that both the charging-based and primal-dual analyses are of interest. Our charging-based analysis is very clean. It was precisely for this reason that we were able to establish the result on MOBVC first and "reverse-engineer" a primal-dual analysis which is, in contrast, somewhat complicated. In retrospect, without the charging analysis, we probably would not be able to come 
up with the primal-dual analysis or even to realize that these problems admit $1+\alpha$ approximation. Nonetheless, the primal-dual analysis is still important since it implies an interesting result on MOBM.

\subsection{The algorithm}

The design of our algorithm for MOBVC is in the same spirit as OBVC. In fact, the major modification needed is to replace $\sum_{u \in N(v)} \max \left\{a-y_{u}, 0\right\}$ by $\hat{f}\left(\mathbf{y}^{\prime}\right)-\hat{f}(\mathbf{y})$ as now the objective function on $L$ is $\hat{f}(\mathbf{y})$ rather than $\sum_{u \in L} y_{u}$.

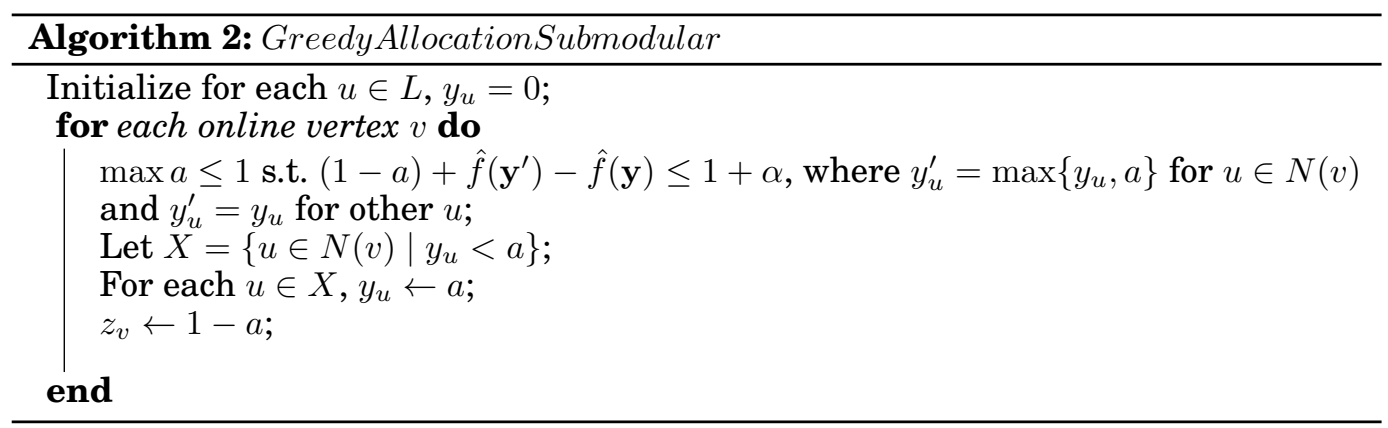

The analysis of GreedyAllocation Submodular makes extensive use of the bar chart representation introduced in Section 2.2.1. It is thus helpful to interpret our algorithm in terms of the bar chart. This will hopefully also make the change in the Lovasz extension $\hat{f}\left(\mathbf{y}^{\prime}\right)-\hat{f}(\mathbf{y})$ more intuitive and easier to visualize.

4.1.1. Bar chart interpretation of the algorithm. We take a closer look at how the bar chart changes after processing an online vertex. Recall that

$$
L(t)=\left\{u \in L \mid y_{u} \geq t\right\}
$$

and $f(L(t))$ is the height of the bar chart at $t$. First of all, observe that for the bars at $t \leq a$, the height changes from $f(L(t))$ to $f(L(t) \cup X)$ since the potential of the vertices from $X$ increased to $a$ and no other vertex increased in potential. As a result, the bar at $t>a$ remains at the same height.

With this observation in mind, we see that a new rectangular region (possibly empty) of height $f(L(t) \cup X)-f(L(t))$ is added to the top of the bar at $t<a$. Moreover, the bar at $t=a$ is effectively split into two $0^{3}$ : the right one has the same height $f(L(a))$ whereas the left one has a larger height $f(L(a) \cup X) \geq f(L(a))$.

Our charging scheme in the next section makes critical use of these two properties:

- All the new rectangular regions are added to the bars at $t \leq a$.

- The total area of the new rectangular regions is $\hat{f}\left(\mathbf{y}^{\prime}\right)-\hat{f}(\mathbf{y})$.

The mechanism in which $1-a$ is charged to $u \in X$ lies in the heart of the previous charging scheme for OBVC. This idea does not quite work anymore as our objective function is submodular rather than modular. The key insight in our new analysis is to charge $1-a$ to the new rectangular regions of the bar chart. This is in contrast to the previous scheme which charges to individual $u \in X$. Towards the end of the next section, we will explain how it is actually a simplistic version of our new scheme.

${ }^{3}$ It is possible to have the degenerate case where $a$ coincides with the boundary of a bar. 
Our analysis in a nutshell is a careful study of figure 2 . The red regions are the new rectangles added to the bar chart. Note that the first three bars increased in height with the third one being split into two at $a$. All of the new regions are found at $t \leq a$. It is no coincidence that the height of the red rectangles decreases along the horizontal axis. Although not needed for the proof, it is instructive to check that this phenomenon is an artifact of submodularity and monotonicity.

In the next section, we propose a charging scheme in which the red new regions are charged to compensate for $z_{v}=1-a$.

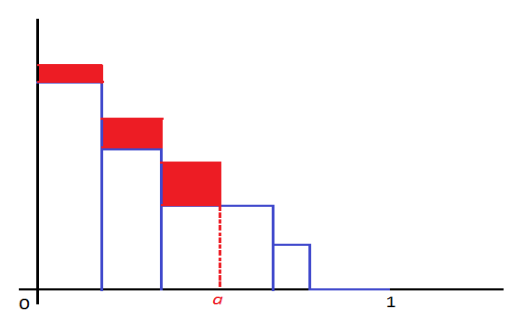

Fig. 2. Bar chart being split at $a$

Finally, we remark that the bar chart is just a pictorial representation of the Lovasz extension. We could have carried out the analysis without it at the expense of added notational complexity. It is for the same reason that various degenerate cases are deemphasized (e.g. we speak of the bar at $t$ but $t$ can happen to be at the boundary between two consecutive bars).

\subsection{Charging-based analysis}

When an online vertex not in the optimal cover is processed, we will charge all the potential used on this vertex to its neighbors, which must be in the optimal cover. More concretely, we charge the cost to the bar chart representing $\hat{f}(\cdot)$. For each point $(x, y)$ of the bar chart, the charging density is $\frac{1-x}{x+\alpha}$. We first show that such charging density is sufficient to account for the potential of the online vertex.

LEMMA 4.1. Let $B$ and $B^{\prime}$ be the bar charts before and after processing online vertex $v$. Let a be the final water-level on the neighbors of $v$ after processing $v$. We have

$$
\int_{B^{\prime} \backslash B} \frac{1-x}{x+\alpha} \mathrm{d} A \geq 1-a .
$$

Proof. The main idea is to charge $1-a$ to the new region of the bar chart. From the discussion in the last section, all the new regions have $x$-coordinates at most $a$. Therefore, we have

$$
\int_{B^{\prime} \backslash B} \frac{1-x}{x+\alpha} \mathrm{d} A \geq \frac{1-a}{a+\alpha} \int_{B^{\prime} \backslash B} \mathrm{~d} A=\frac{1-a}{a+\alpha} \cdot\left(\hat{f}\left(\mathbf{y}^{\prime}\right)-\hat{f}(\mathbf{y})\right)=1-a,
$$

where the last equality obviously holds if $a=1$. If $a<1$, then we must have exhausted all of our resources $1+\alpha$ (otherwise $a$ would be larger) and hence we have $\hat{f}\left(\mathbf{y}^{\prime}\right)-\hat{f}(\mathbf{y})=a+\alpha$.

Now we show that the total charges to the left vertices by online vertices not in the optimal cover $C^{*}$ is at most $\alpha \cdot f\left(L \cap C^{*}\right)$.

LEMMA 4.2. The total charges received from online vertices $R \backslash C^{*}$ is $\leq \alpha \cdot f\left(L \cap C^{*}\right)$. 
PROOF. Let $B^{*}$ be the union of the new regions in the bar chart generated by processing online vertices $R \backslash C^{*}$. Therefore, the total charges are

$$
\int_{B^{*}} \frac{1-x}{x+\alpha} \mathrm{d} A \text {. }
$$

For $t \in[0,1]$, let $B^{*}(t)$ be the intersection of $B^{*}$ with the line $x=t$. We have

$$
\int_{B^{*}} \frac{1-x}{x+\alpha} \mathrm{d} A=\int_{0}^{1} \int_{B^{*}(x)} \frac{1-x}{x+\alpha} \mathrm{d} y \mathrm{~d} x \leq \int_{0}^{1} \frac{1-x}{x+\alpha} \mathrm{d} x \sup _{t \in[0,1]} \int_{B^{*}(t)} \mathrm{d} y=\alpha \cdot \sup _{t \in[0,1]} \int_{B^{*}(t)} \mathrm{d} y .
$$

It is then sufficient to show that for $t \in[0,1]$,

$$
\int_{B^{*}(t)} \mathrm{d} y \leq f\left(L \cap C^{*}\right) .
$$

Notice that $\int_{B^{*}(t)} \mathrm{d} y$ is the total height of regions added to the bar chart at $x=t$ when processing online vertices in $R \backslash C^{*}$.

Although the proof below looks somewhat technical, the key idea is simple. Suppose that all of the vertices in $L \backslash C^{*}$ are removed, i.e. $L \subseteq C^{*}$. Now the height of the bar chart is at most $f(L)=f\left(C^{*} \cap L\right)$ so our claim is clear. If we add back $L \backslash C^{*}$, recall that we care only about the rectangles added for $v \notin C^{*}$. The height of the additional rectangle is just the marginal difference, which cannot be worse than before because of diminishing marginal return. We formalize this below.

Let $L_{i}(t)$ be the set $L(t)=\left\{u \in L \mid y_{u} \geq t\right\}$ after processing the $i$-th online vertex $v_{i}$. Since $y_{u}$ can never decrease for all $u \in L$, we have

$$
L_{0}(t) \subseteq L_{1}(t) \subseteq \cdots \subseteq L_{|R|}(t) .
$$

Furthermore, $L_{i}(t) \backslash L_{i-1}(t) \subseteq N\left(v_{i}\right)$ since only $y_{u}$ for $u \in N\left(v_{i}\right)$ can increase when processing $v_{i}$. In particular, for $v_{i} \notin C^{*}$ we have that $L_{i}(t) \backslash L_{i-1}(t) \subseteq C^{*}$ as $v_{i} \notin C^{*}$ implies $N\left(v_{i}\right) \subseteq C^{*}$. Submodularity and $L_{i}(t) \backslash L_{i-1}(t) \subseteq C^{*}$ for $v_{i} \notin C^{*}$ give

$$
f\left(L_{i}(t)\right)-f\left(L_{i-1}(t)\right) \leq f\left(L_{i}(t) \cap C^{*}\right)-f\left(L_{i-1}(t) \cap C^{*}\right) .
$$

Finally, when processing $v_{i}$, the height of the new rectangular region ${ }^{4}$ at $t$ is precisely $f\left(L_{i}(t)\right)-f\left(L_{i-1}(t)\right)$. Now the sum of the height of the rectangular regions at $t$ added when processing $v_{i} \notin C^{*}$ is

$$
\begin{array}{rlr}
\int_{B^{*}(t)} \mathrm{d} y & =\sum_{v_{i} \in R \backslash C^{*}} f\left(L_{i}(t)\right)-f\left(L_{i-1}(t)\right) & \\
& \leq \sum_{v_{i} \in R \backslash C^{*}} f\left(L_{i}(t) \cap C^{*}\right)-f\left(L_{i-1}(t) \cap C^{*}\right) \quad \text { (submodularity) } \\
& \leq \sum_{i=1}^{|R|} f\left(L_{i}(t) \cap C^{*}\right)-f\left(L_{i-1}(t) \cap C^{*}\right) & \text { (monotonicity) } \\
& =f\left(L_{|R|}(t) \cap C^{*}\right)-f\left(L_{0}(t) \cap C^{*}\right) \leq f\left(L \cap C^{*}\right) .
\end{array}
$$

Here the last inequality follows from monotonicity and non-negativeness of $f$.

LEMMA 4.3. The total resources used in processing online vertices $R \backslash C^{*}$ are at most $(1+\alpha) \cdot f\left(L \cap C^{*}\right)$.

\footnotetext{
${ }^{4}$ Of course, it is possible that no region is added in which case this is still okay as $f\left(L_{i}(t)\right)=f\left(L_{i-1}(t)\right)$.
} 
Proof. For the $i$-th online vertex $v_{i} \in R$, we define $\mathbf{y}_{i}$ to be the vector of potentials on $L$ after processing $v_{i}$. Then, by our algorithm and the last lemma, the total resources used in processing $R \backslash C^{*}$ are at most

$$
\alpha \cdot f\left(L \cap C^{*}\right)+\sum_{v_{i} \in R \backslash C^{*}} \hat{f}\left(\mathbf{y}_{i}\right)-\hat{f}\left(\mathbf{y}_{i-1}\right) .
$$

Since for $v_{i} \in R \backslash C^{*}, L_{i}(t) \backslash L_{i-1}(t) \subseteq C^{*}$ for any $t \in[0,1]$, where $L_{i}(t)$ is defined as before. By Eqn.(2) and the definition of $\hat{f}(\cdot)$, we have

$$
\begin{aligned}
\sum_{v_{i} \in R \backslash C^{*}} \hat{f}\left(\mathbf{y}_{i}\right)-\hat{f}\left(\mathbf{y}_{i-1}\right) & \leq \sum_{v_{i} \in R \backslash C^{*}} \hat{f}\left(\left.\mathbf{y}_{i}\right|_{L \cap C^{*}}\right)-\hat{f}\left(\left.\mathbf{y}_{i-1}\right|_{L \cap C^{*}}\right) \\
& \leq \sum_{v_{i} \in R} \hat{f}\left(\left.\mathbf{y}_{i}\right|_{L \cap C^{*}}\right)-\hat{f}\left(\left.\mathbf{y}_{i-1}\right|_{L \cap C^{*}}\right) \\
& =\hat{f}\left(\left.\mathbf{y}_{|R|}\right|_{L \cap C^{*}}\right)-\hat{f}\left(\left.0\right|_{L \cap C^{*}}\right) \leq f\left(L \cap C^{*}\right),
\end{aligned}
$$

where $\left.\mathbf{y}_{i}\right|_{L \cap C^{*}}$ restricts the vector $\mathbf{y}_{i}$ to the vertices $L \cap C^{*}$ by setting the other entries to 0 . This concludes the proof.

Therefore, our algorithm uses resources at most $(1+\alpha) \cdot f\left(L \cap C^{*}\right)$ when processing vertices in $R \backslash C^{*}$. On the other hand, it uses resources at most $(1+\alpha) \cdot\left|R \cap C^{*}\right|$ for other online vertices as processing each of them increased the total potentials by at most $1+\alpha$. Our algorithm thus $1+\alpha$-competitive for the fractional matroid online bipartite vertex cover problem. Since we can always round a fractional solution to a randomized integral solution (section 2.4), we have the following theorem.

THEOREM 4.4. There exists an optimal $1+\alpha$-competitive algorithm for the matroid online bipartite integral vertex cover problem.

\subsection{Primal-dual analysis}

We first review the key ingredients used in the original primal-dual analysis of online bipartite matching in [Buchbinder et al. 2007], which largely consists of two steps:

- Employs such constraints as $x_{u}=g\left(y_{u}\right)$ (or $x_{u} \leq g\left(y_{u}\right)$ ) for some suitable increasing function $g$. The motivation for doing this is to enforce some correlation between the primal and dual variables so that, for instance, when $x_{u}$ is small, $y_{u}$ is not too big which allows room to pay for the future increase in $x_{u}$.

- Relates the size of the primal and dual solutions by $\sum\left(g(a)-g\left(y_{u}\right)\right) \approx c(1-$ $\left.a+\sum\left(a-y_{u}\right)\right)$ for some constant $c$. As in the usual primal-dual method, this is essential for bounding the size of the solution via weak duality.

This scheme depends crucially on the fact that the cost function is modular. For submodular cost functions, one may try to imitate that by using constraints like $x_{S} \leq f(S) g(h(\mathbf{y} \mid S))(\mathbf{y} \mid S$ is the vector restricted to $S)$, where $g$ is the same as before and $h:[0,1]^{S} \longrightarrow[0,1]$ is some suitable function.

Considering the Lovasz extension, the most natural choice is probably $h(\mathbf{y} \mid S)=$ $\min _{u \in S} y_{u}$. But this is fundamentally flawed as one may have a very small $y_{u}$ with other $y_{u^{\prime}}=1$. It turns out that, perhaps somewhat counter-intuitively, the correct function is $h(\mathbf{y} \mid S)=\max _{u \in S} y_{u}$.

Even more surprisingly, the constraint $x_{S} \leq f(S) g(h(\mathbf{y} \mid S))$ alone is not enough to relate the cost of the primal and dual solutions. Recall that $\hat{f}(\mathbf{y})=\sum f\left(Y_{i}\right)\left(y_{i}-y_{i-1}\right)$ for a fixed ordering of $y$. Thus one might hope to consider $S=Y_{1}, Y_{2}, \ldots$ in order to relate the increment in the size of the primal and dual solutions. Unfortunately, this does not work as the ordering of $y$ typically changes over the execution of the algorithm. 
To rescue this, we turn to the bar chart representation again. Instead of one global ordering, a local ordering is imposed on each bar of the bar chart. More precisely, for a bar at $t$, we maintain an ordering $\sigma_{t}$ of its existing vertices $L(t)$. When $L(t)$ increases, we extend the current ordering by arbitrarily appending the new vertices to its end. We formalize our ideas in the rest of this section.

To simplify our notation, we view $x_{u}$ as a function on $[0,1]$ and the value of $x_{u}{ }^{5}$ is

$$
x_{u}=\int_{0}^{1} x_{u}(t) d t .
$$

This perspective will be useful when we analyze our algorithm using the bar chart representation (which can be seen as a function on $[0,1]$ ). The $x_{u}$ produced by the algorithm will be a piecewise constant function. Conceptually, $\int_{0}^{1} x_{u}(t) d t$ aggregates over the contribution of each bar to $x_{u}$.

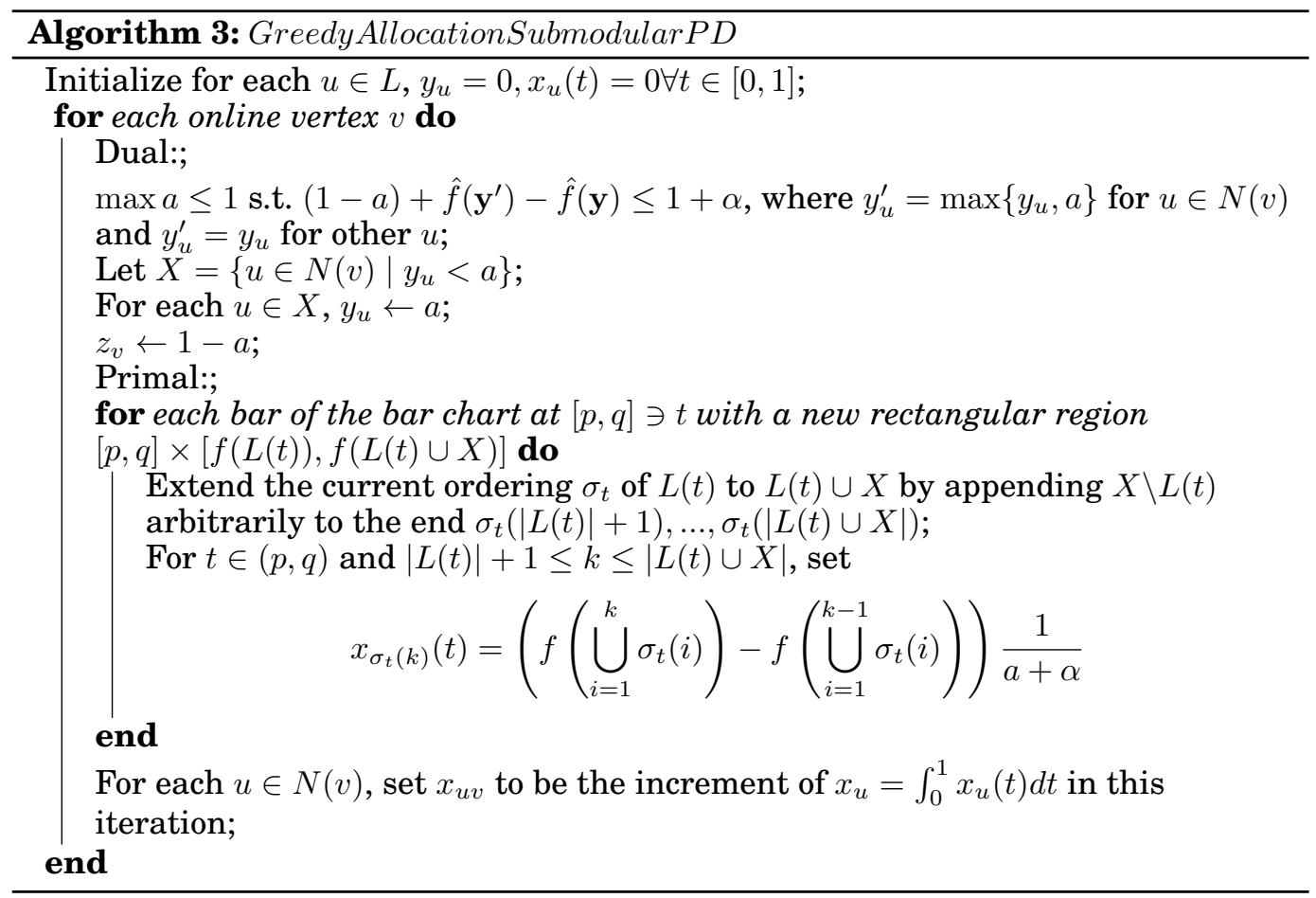

At the first glance, our primal update seems somewhat convoluted. The underlying philosophy is nevertheless much simpler. Before proceeding to the analysis, we first unpack the details of the algorithm along with some simple observations.

First of all, in our algorithm we focus on $x_{u}(t)$ rather than $x_{u v}$. This is more convenient in the analysis since what matters is the extent to which $u$ is matched (recall: $x_{S} \leq f(S)$ ) but not which edge is assigned to $u$. Thus in the algorithm, we determine only how much $x_{u}$ increases and retroactively what $x_{u v}$ is.

\footnotetext{
${ }^{5}$ We abuse notations by using $x_{u}$ for both the primal variable as well as a function on $[0,1]$.
} 
Note that since each vertex can be added at most once to $L(t), x_{u}(t)$ can increase at most once and this increment will be from $x_{u}(t)=0$ to $x_{u}(t)=$ $\left(f\left(\bigcup_{i=1}^{k} \sigma_{t}(i)\right)-f\left(\bigcup_{i=1}^{k-1} \sigma_{t}(i)\right)\right) \frac{1}{a+\alpha}$, where $u=\sigma_{t}(k)$.

Lastly, we emphasize the role of the ordering $\sigma_{t}$. This is the key ingredient that makes the analysis possible. See Proposition 4.5 and Lemma 4.6 for more details.

We are now ready to analyze the algorithm. There are three major components:

- (feasibility) $x_{S} \leq f(S)$ for all $S \subseteq L$.

- (feasibility) $x_{v} \leq 1$, i.e. the total increment of all $x_{u}$ in each iteration is at most 1 .

- (competitiveness) $\triangle D=(1+\alpha) \triangle P$, where $\triangle D$ and $\triangle P$ are the increments in the size of the dual and primal solutions respectively.

Once the above have been established, we can conclude that our algorithm is correct and achieves a competitive ratio of $1+\alpha$ via weak duality.

The following well-known property of the base polyhedron will be used in the analysis. For completeness a proof is given in the full version.

Proposition 4.5. Let $f: \Omega \longrightarrow \mathbb{R}$ be a monotone submodular function and fix an ordering $\sigma:\{1,2, \ldots,|\Omega|\} \longrightarrow \Omega$. Then the solution

$$
x_{\sigma(k)}=f\left(\bigcup_{i=1}^{k} \sigma(i)\right)-f\left(\bigcup_{i=1}^{k-1} \sigma(i)\right)
$$

satisfies the inequalities $x_{S} \leq f(S) \forall S \subseteq \Omega$.

LEMMA 4.6. In GreedyAllocationSubmodularPD, we have $x_{S} \leq f(S)$ for all $S \subseteq L$.

Proof. We first show that for each $t$,

$$
x_{S}(t) \leq \frac{f(S)}{t+\alpha}
$$

Consider any $\sigma_{t}(k) \in S$ for which $x_{\sigma_{t}(k)}(t)>0$. Then we must have set

$x_{\sigma_{t}(k)}(t)=\left(f\left(\bigcup_{i=1}^{k} \sigma_{t}(i)\right)-f\left(\bigcup_{i=1}^{k-1} \sigma_{t}(i)\right)\right) \frac{1}{a+\alpha} \leq\left(f\left(\bigcup_{i=1}^{k} \sigma_{t}(i)\right)-f\left(\bigcup_{i=1}^{k-1} \sigma_{t}(i)\right)\right) \frac{1}{t+\alpha}$,

where the inequality follows from the fact that only the bars on the left of $a$ increase in height and hence $t \leq a$.

Now by Proposition 4.5, we have $x_{S}(t) \leq \frac{f(S)}{t+\alpha}$. Our desired result thus follows:

$$
x_{S}=\int_{0}^{1} x_{S}(t) d t \leq \int_{0}^{1} \frac{f(S)}{t+\alpha} d t=f(S) .
$$

LEMMA 4.7. For each iteration of the algorithm, the increases in the size of the primal and dual solutions satisfy

$$
\triangle D=(1+\alpha) \triangle P
$$

PROOF. Full version.

COROLlaRY 4.8. $x_{v} \leq 1$, i.e. the total increment of all $x_{u}$ in each iteration is $\leq 1$.

Proof. The dual solution can increase by at most $1+\alpha$ and hence $\triangle P$, which is just $x_{v}$, is at most 1 by Lemma 4.7 . 
Combining all the pieces, we obtain our main theorem.

THEOREM 4.9. Our algorithm is $1-1 /$ e-competitive for matroid online bipartite matching and $1+\alpha$-competitive for matroid online bipartite vertex cover.

Proof. By Lemma 4.7, we always have $D=(1+\alpha) \cdot P$. By weak duality (see Lemma 2.1), we can bound $P$ and $D$ against the optimal solutions $D^{*}$ and $P^{*}$ as follows,

$$
P^{*} \leq D=(1+\alpha) \cdot P \leq(1+\alpha) \cdot D^{*} .
$$

This shows that $P \geq(1-1 / e) P^{*}$ and $D \leq(1+\alpha) D^{*}$, as desired.

Finally, we remark that we do have $x_{S} \leq f(S) \frac{\max _{u \in S} y_{u}+\int_{0}^{y_{u}} \frac{1-t}{t+\alpha} d t}{1+\alpha}$ (i.e. $x_{S} \leq$ $\left.f(S) g\left(\max _{u \in S} y_{u}\right)\right)$ as mentioned earlier. Although not needed for the proof, it has served as a useful inspiration when we were developing this primal-dual analysis.

\subsection{Extensions}

We briefly discuss extensions of our techniques to other problems related to online bipartite matching. Using similar machineries, it is rather straightforward to generalize the adwords [Mehta et al. 2007; Buchbinder et al. 2007] and online ad assignment problems to the matroid setting [Feldman et al. 2009a; Devanur et al. 2013a]. We however do not discuss the details to avoid being repetitive.

For Matroid Adwords, by considering the following primal and dual programs one can argue that the waterfilling algorithm is again $1-1 / e$-competitive.

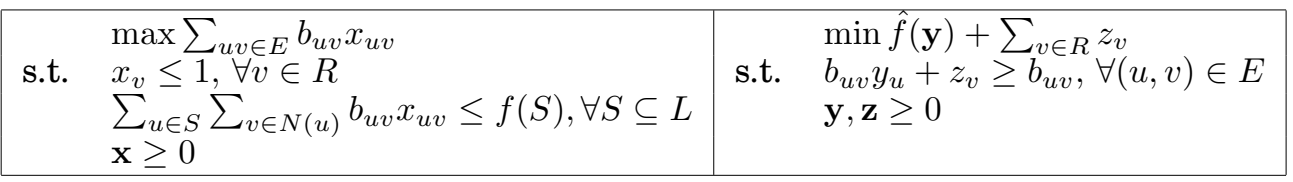

\begin{tabular}{|c|c|c|c|}
\hline s.t. & $\begin{array}{l}\max \sum_{e \in E} w_{e} x_{e} \\
x_{v} \leq 1, \forall v \in R \\
x_{S} \leq f(S), \forall S \subseteq L\end{array}$ & & $\begin{array}{l}\min \hat{f}(\mathbf{y})+\sum_{v \in R} z_{v} \\
y_{u}+z_{v} \geq w_{u v}, \forall(u, v) \in E \\
\mathbf{y}, \mathbf{z} \geq 0\end{array}$ \\
\hline
\end{tabular}

For matroid online ad assignment one would consider these programs instead:

\section{MATROID ONLINE BIPARTITE MATCHING IN THE RANDOM ARRIVAL MODEL}

It is known that Greedy is $1-1$ /e-competitive for Online Bipartite Matching in the random arrival model (without the small bid assumption) [Goel and Mehta 2008]. In this section, we combine the randomized primal-dual analysis of [Devanur et al. 2013b] with our convex program to prove that Greedy remains $1-1 / e$-competitive even for Matroid Online Bipartite Matching. Recall that unlike the last section we are now working with the integral version and $f$ is a matroid rank function.

\subsection{Review of Randomized Primal-Dual Analysis}

The elegant paper of [Devanur et al. 2013b] introduced the randomized primal-dual analysis, a clever yet simple extension of standard primal-dual. One of the drawbacks of the standard primal-dual analysis is that it is typically hard to accommodate for randomized algorithms, since very often one has both dual feasibility and bounded duality gap at all time. These patterns render primal-dual style analyses of most randomized algorithms seemingly impossible. To get around with the issue, randomized primal-dual requires only dual feasibility in expectation (while still having bounded duality gap at all time). This is sufficient because of linearity of expectation. 


\subsection{Randomized Primal-Dual Analysis of Greedy for MOBM}

Greedy is a natural algorithm for (Matroid) Online Bipartite Matching where we simply match a new online vertex $v$ to an available offline vertex according to some fixed preference ordering $\sigma^{(v)}$. Greedy is known to be $1-1 / e$-competitive [Goel and Mehta 2008 ] and in this section, we generalize this result to MOBM.

Inspired by [Devanur et al. 2013b], we present a randomized primal-dual analysis of Greedy. Let $M_{L} \subseteq L$ be the set of matched vertices and $\operatorname{span}\left(M_{L}\right)=\{u \in L$ : $\left.f\left(M_{L}+u\right)=f\left(M_{L}\right)\right\}$ be the span of $M_{L}$ w.r.t. the matroid given by $f$. Note that a vertex $u \in L$ can still be matched iff $u \notin \operatorname{span}\left(M_{L}\right)$, which we therefore should keep track of.

Recall that we are working with the random arrival model where online vertices arrive in a uniformly random order. Equivalently, we may sample $t_{v} \in[0,1]$ for $v \in R$ and make them arrive in ascending order prescribed by $t_{v}$. This parameterization is key to randomly setting the dual variables $y_{u}, z_{v}$. Let $g(t)=e^{t-1}$.

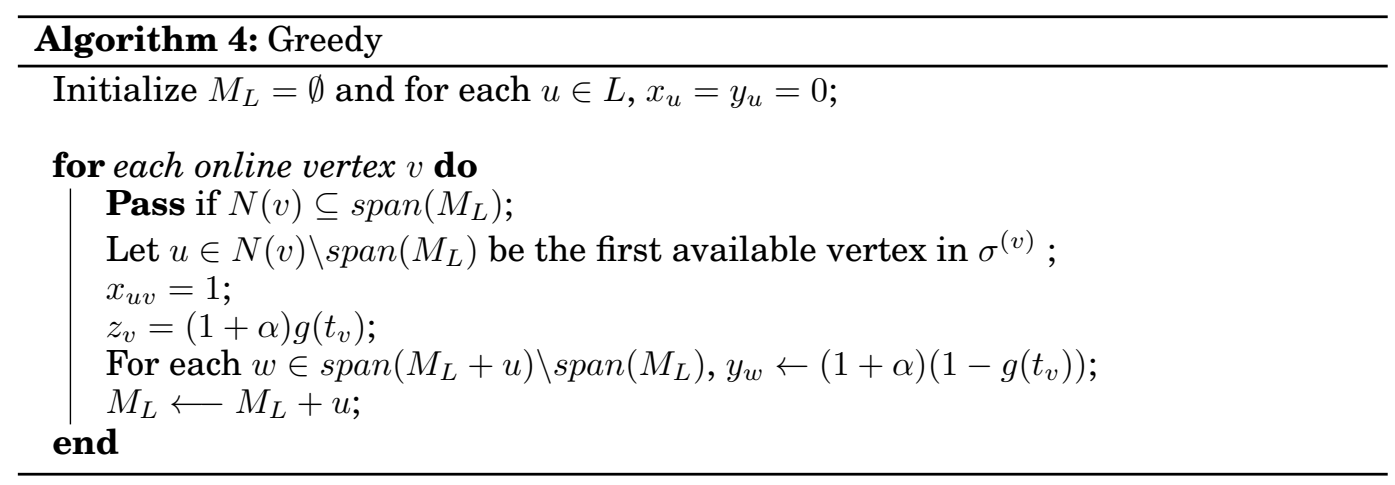

It is clear that the matching maintained by the algorithm is valid. To prove that Greedy works, we need to establish (expected) dual feasibility and duality gap.

Observe that by design each $w \in L$ is updated at most once. Moreover, every $w \in$ $\operatorname{span}\left(M_{L}\right)$ must have been updated.

LEMMA 5.1 (DUALITY GAP). For each iteration of the algorithm, the increases in the size of the primal and dual solutions satisfy

$$
\triangle D=(1+\alpha) \triangle P \text {. }
$$

Proof. If no vertex is matched, $\triangle D=\triangle P=0$ and the result follows. Otherwise, $\triangle P=x_{u v}=1$ and we claim that $\triangle D=1+\alpha$. Let $y^{\prime}$ be the new $y$.

First of all,

$$
\triangle D=z_{v}+\hat{f}\left(y^{\prime}\right)-\hat{f}(y)=(1+\alpha) g\left(t_{v}\right)+\hat{f}\left(y^{\prime}\right)-\hat{f}(y)
$$

so it suffices to show $\hat{f}\left(y^{\prime}\right)-\hat{f}(y)=(1+\alpha)\left(1-g\left(t_{v}\right)\right)$. We make two observations: $y^{\prime}$ and $y$ differ exactly in $\operatorname{span}\left(M_{L}+u\right) \backslash \operatorname{span}\left(M_{L}\right)$ and that for $w^{\prime} \in \operatorname{span}\left(M_{L}\right), y_{w^{\prime}} \geq$ $(1+\alpha)\left(1-g\left(t_{v}\right)\right)$. The former is clear while the latter follows from the fact that $w^{\prime}$ was updated before $v$ arrives and hence the online vertex $v^{\prime}$ used to update $w^{\prime}$ must have

$$
t_{v^{\prime}} \leq t_{v} \Longrightarrow y_{w^{\prime}}=(1+\alpha)\left(1-g\left(t_{v^{\prime}}\right)\right) \geq(1+\alpha)\left(1-g\left(t_{v}\right)\right) .
$$

In other words, all the new coordinates in $y^{\prime}$ have values not greater than the existing ones. Now $\hat{f}\left(y^{\prime}\right)-\hat{f}(y)=(1+\alpha)\left(1-g\left(t_{v}\right)\right)$ holds because $f\left(\operatorname{span}\left(M_{L}+u\right)\right)-$ $f\left(\operatorname{span}\left(M_{L}\right)\right)=1$ (recall that $f$ is the rank function of the matroid). 
Next we show that the dual is feasible in expectation, i.e. $\mathbb{E}\left[y_{w}\right]+\mathbb{E}\left[z_{v}\right] \geq 1$ for any edge $w v$. For this we need the notion of critical values. Suppose that we run Greedy on $G$ without $v$ and let $w$ be updated when an online vertex of random value $t^{c} \in[0,1]$ arrives (if $w$ is never updated, $t^{c}=1$ ). We have the following two lemmas:

LEMma 5.2 (DOMINANCE). Given $t_{v^{\prime}}$ for $v^{\prime} \neq v$, $v$ is matched whenever $t_{v}<t^{c}$.

PROOF. If $t_{v}<t^{c}$, then $w$ must not have been updated when $v$ arrives. Furthermore, $w$ is not in the current $\operatorname{span}\left(M_{L}\right)$ as it is updated only after in the run without $v$. Therefore when $v$ arrives, $w$ is available and $v$ can be matched (possibly to a vertex other than $w$ ).

LEMma 5.3 (MONOTONICITY). Given $t_{v^{\prime}}$ for $v^{\prime} \neq v, y_{w} \geq(1+\alpha)\left(1-g\left(t^{c}\right)\right)$ regardless of the value of $t_{v}$.

Proof. Let $M_{L}$ and $M_{L}^{c}$ be the set of matched vertices in the run with and without $v$ respectively. It is easy to show by induction that $\operatorname{span}\left(M_{L}^{c}\right) \subseteq \operatorname{span}\left(M_{L}\right)$ at all time. In particular, $w$ can only be updated earlier in the run with $v$. In other words, the value $t$ used to update $w$ is either the same or smaller, as desired.

LEMMA 5.4 (DUAL FEASIBILITY). We have $\mathbb{E}\left[y_{w}\right]+\mathbb{E}\left[z_{v}\right] \geq 1$ for any edge wv.

Proof. Let $t_{-v}$ denote the random values other than $t_{v}$. By the previous two lemmas, $\mathbb{E}\left[z_{v} \mid t_{-v}\right] \geq \int_{0}^{t^{c}}(1+\alpha) g(t) d t$ and $\mathbb{E}\left[y_{w} \mid t_{-v}\right] \geq(1+\alpha)\left(1-g\left(t^{c}\right)\right)$. Therefore

$$
\mathbb{E}\left[y_{w} \mid t_{-v}\right]+\mathbb{E}\left[z_{v} \mid t_{-v}\right] \geq(1+\alpha)\left(1-g\left(t^{c}\right)\right)+\int_{0}^{t^{c}}(1+\alpha) g(t) d t=1
$$

where we used $g(t)=e^{t-1}$ for direct calculation. Now our result follows by taking expectation over $t_{-v}$.

Combining the previous lemmas, we obtain:

THEOREM 5.5. Greedy is 1-1/e-competitive for matroid online bipartite matching in the random arrival model.

\section{DISCUSSION AND FUTURE RESEARCH}

Our work naturally raises numerous questions about online algorithms. We have selected three of them which we find the most interesting.

- Submodularity in other online problems. In online algorithms, submodularity seems to be considered less often than other themes in combinatorial optimization. We hope that our work will stimulate the interest in combining submodularity with existing online problems in the literature. Our results on MOBM and MOBVC show that various ingredients used in offline submodular optimization are still applicable online. We are hopeful that some of the powerful machineries developed for submodularity over the past few decades will find applications in various online settings.

- Two-sided submodular. In both MOBM and MOBVC, a submodular function $f$ is imposed on the left vertices $L$. A natural extension is to impose also a submodular function $g$ on the right vertices $R$. It is not hard to see that $1+\alpha$ cannot be attained anymore for both problems. Nevertheless, the corresponding offline problems are still solvable in polynomial time and do exhibit many nice typical properties such as the existence of integral optimal solution. In light of this, we believe that some constant approximation would still be achievable.

- Integral MOBM. Can we remove the small bid assumption needed for the waterfilling algorithm? We conjecture that this algorithm works for the special case of 
partition matroids: randomly permute each partition and the vertices within each partition; match according to this ordering. This algorithm generalizes the classical algorithm RANKING [Karp et al. 1990] in two ways: treating each offline vertex as a singleton partition or treating the entire $L$ as one partition.

\section{REFERENCES}

G. Aggarwal, G. Goel, C. Karande, and A. Mehta. 2011. Online vertex-weighted bipartite matching and single-bid budgeted allocations. In Proceedings of the Twenty-Second Annual ACM-SIAM Symposium on Discrete Algorithms. SIAM, 1253-1264.

N. Bansal, A. Gupta, J. Li, J. Mestre, V. Nagarajan, and A. Rudra. 2010. When LP is the cure for your matching woes: improved bounds for stochastic matchings. Algorithms-ESA 2010 (2010), 218-229.

A. Blum, T. Sandholm, and M. Zinkevich. 2006. Online algorithms for market clearing. Journal of the ACM (JACM) 53, 5 (2006), 845-879.

N. Buchbinder, K. Jain, and J. Naor. 2007. Online primal-dual algorithms for maximizing ad-auctions revenue. Algorithms-ESA 2007 (2007), 253-264.

N.R. Devanur and K. Jain. 2012. Online matching with concave returns. In Proceedings of the 44th symposium on Theory of Computing. ACM, 137-144.

N.R. Devanur, K. Jain, and R.D. Kleinberg. 2013b. Randomized Primal-Dual analysis of RANKING for Online Bipartite Matching. In SODA '13: Proceedings of the thirteenth Annual ACM-SIAM Symposium on Discrete Algorithms. to appear.

Nikhil R Devanur, Zhiyi Huang, Nitish Korula, Vahab S Mirrokni, and Qiqi Yan. 2013a. Whole-page optimization and submodular welfare maximization with online bidders. In Proceedings of the fourteenth ACM conference on Electronic commerce. ACM, 305-322.

Nikhil R Devanur, Balasubramanian Sivan, and Yossi Azar. 2012. Asymptotically optimal algorithm for stochastic adwords. In Proceedings of the 13th ACM Conference on Electronic Commerce. ACM, 388404.

Nikhil R. Devenur and Thomas P. Hayes. 2009a. The adwords problem: online keyword matching with budgeted bidders under random permutations. In EC '09: Proceedings of the tenth ACM conference on Electronic commerce. ACM, New York, NY, USA, 71-78. DOI : http://dx.doi.org/10.1145/1566374.1566384

Nikhil R Devenur and Thomas P Hayes. 2009b. The adwords problem: online keyword matching with budgeted bidders under random permutations. In Proceedings of the 10th ACM conference on Electronic commerce. ACM, 71-78.

Jon Feldman, Nitish Korula, Vahab Mirrokni, S Muthukrishnan, and Martin Pál. 2009a. Online ad assignment with free disposal. In Internet and Network Economics. Springer, 374-385.

J. Feldman, A. Mehta, V. Mirrokni, and S. Muthukrishnan. 2009b. Online stochastic matching: Beating 1-1/e. In Foundations of Computer Science, 2009. FOCS'09. 50th Annual IEEE Symposium on. IEEE, $117-126$.

Gagan Goel and Aranyak Mehta. 2008. Online budgeted matching in random input models with applications to Adwords. In SODA '08: Proceedings of the nineteenth annual ACM-SIAM symposium on Discrete algorithms. Society for Industrial and Applied Mathematics, Philadelphia, PA, USA, 982-991.

B. Kalyanasundaram and K.R. Pruhs. 2000. An optimal deterministic algorithm for online b-matching. Theoretical Computer Science 233, 1 (2000), 319-325.

C. Karande, A. Mehta, and P. Tripathi. 2011. Online bipartite matching with unknown distributions. In Proceedings of the 43rd annual ACM symposium on Theory of computing. ACM, 587-596.

R.M. Karp, U.V. Vazirani, and V.V. Vazirani. 1990. An optimal algorithm for on-line bipartite matching. In Proceedings of the twenty-second annual ACM symposium on Theory of computing. ACM, 352-358.

M. Mahdian and Q. Yan. 2011. Online bipartite matching with random arrivals: an approach based on strongly factor-revealing lps. In Proceedings of the 43rd annual ACM symposium on Theory of computing. ACM, 597-606.

V.H. Manshadi, S.O. Gharan, and A. Saberi. 2011. Online stochastic matching: Online actions based on offline statistics. In Proceedings of the Twenty-Second Annual ACM-SIAM Symposium on Discrete Algorithms. SIAM, 1285-1294.

A. Mehta, A. Saberi, U. Vazirani, and V. Vazirani. 2007. Adwords and generalized online matching. Journal of the ACM (JACM) 54, 5 (2007), 22.

Yajun Wang and Sam Chiu-wai Wong. 2015. Two-sided online bipartite matching and vertex cover: Beating the greedy algorithm. In Automata, Languages, and Programming. Springer, 1070-1081. 